TRANSACTIONS OF THE

AMERICAN MATHEMATICAL SOCIETY

Volume 350, Number 4, April 1998, Pages 1681-1701

S 0002-9947(98)02002-9

\title{
ALGEBRAIC INVARIANT CURVES FOR THE LIÉNARD EQUATION
}

\author{
HENRYK ŻOŁA̧DEK
}

\begin{abstract}
Odani has shown that if $\operatorname{deg} g \leq \operatorname{deg} f$ then after deleting some trivial cases the polynomial system $\dot{x}=y, \dot{y}=-f(x) y-g(x)$ does not have any algebraic invariant curve. Here we almost completely solve the problem of algebraic invariant curves and algebraic limit cycles of this system for all values of $\operatorname{deg} f$ and $\operatorname{deg} g$. We give also a simple presentation of Yablonsky's example of a quartic limit cycle in a quadratic system.
\end{abstract}

\section{The RESUlts}

The subject of this work is the polynomial Liénard system

$$
\dot{x}=y, \dot{y}=-f_{m}(x) y-g_{n}(x),
$$

where $f_{m}(x)=\sum_{0}^{m} a_{i} x^{i}$ and $g_{n}(x)=\sum_{0}^{n} b_{i} x^{i}$ are polynomials of degrees $m$ and $n$ respectively $\left(a_{m} b_{n} \neq 0\right)$. Odani in [3] investigated algebraic phase curves of (1). He proved that if $n \leq m$ and

$$
f_{m} g_{n}\left(f_{m} / g_{n}\right)^{\prime} \not \equiv 0
$$

then the vector field (1) does not have any algebraic invariant curve.

He also presented the example of Wilson [6] with

$$
f_{2}=\mu\left(x^{2}-1\right), g_{5}=\mu^{2} x^{3}\left(x^{2}-4\right) / 16+x
$$

and the algebraic limit cycle

$$
\left[y+\mu x\left(x^{2}-4\right)\right]^{2}+x^{2}-4=0 .
$$

Odani conjectured that if $m<n<2 m+1$ then (1) does not have algebraic limit cycles. Here we show that this conjecture is false, and we solve the problem of algebraic limit cycles and algebraic invariant curves for all pairs $(m, n)$ almost completely.

In what follows we consider systems of the form (1) with the restriction (2) and denote by $\mathcal{A}_{m, n}$ the space of such systems, $\mathcal{A}_{m, n} \simeq \mathbb{C}^{m+n} \times(\mathbb{C} \backslash 0)^{2}$ for $m \neq n$.

Theorem 1. (a) If $m<n$, then there are systems from $\mathcal{A}_{m, n}$ with an algebraic invariant curve.

(b) If $m<n,(m, n) \neq(0,1)$, then a generic system from $\mathcal{A}_{m, n}$ does not have algebraic invariant curves.

Received by the editors April 10, 1995 and, in revised form, August 26, 1996.

1991 Mathematics Subject Classification. Primary 34C05, 58F21.

Supported by Polish KBN Grant No 2 P03A 02208.

(C) 1998 American Mathematical Society 
(c) If $m>1, n>m+1,(m, n) \neq(2,4)$, then there exist systems from $\mathcal{A}_{m, n}$ with an algebraic limit cycle.

(d) If $m=0$ or $m=1, n \neq 3$ or $m=2, n=4$ or $n=m+1$, then any real system from $\mathcal{A}_{m, n}$ does not have algebraic limit cycles.

During the demostration of this theorem we shall also give another proof of Odani's theorem. We see that for $m=1, n=3$ the problem of algebraic limit cycles remains open.

We can also deduce something about the possible forms of algebraic invariant curves. We say that an algebraic curve $K(x, y)=0$ is $d$-fold if the degree of the polynomial $K$ with respect to $y$ is equal to $d$. (Note that in the next theorem we do not assume that the invariant curve is irreducible).

Theorem 2. If $K(x, y)=0$ is an invariant algebraic curve for a system from $\mathcal{A}_{m, n}$, then we have one of the following three possibilities.

(a) The curve is rational of the form $y=P(x)$, where $P$ is a polynomial (for $m<n \leq 2 m+1$ or $n=2 k-1>2 m+1)$.

(b) The curve is hyperelliptic of the form $(y+P(x))^{2}=Q(x)$, where $P, Q$ are polynomials (for $m<n$ ).

(c) The curve is $d$-fold, $d \geq 3, n=2 m+1$, and the equation

$$
(m+1)\left(4 m+4-a_{m}^{2} / b_{2 m+1}\right) \lambda^{2}-\left(4 m+4-a_{m}^{2} / b_{2 m+1}\right) \lambda+1=0
$$

has only rational solutions $\lambda_{1,2}$.

Remark. If $n \neq 2 m+1$ or $n=2 m+1$ but (4) has irrational solutions, then the hyperelliptic invariant curve is defined uniquely. In these cases there is the upper bound $\left[\frac{m+1}{2}\right]$ for the number of algebraic limit cycles of the Liénard system (1).

Theorem 2 shows some similarities between algebraic Liénard equations and the algebraic Riccati equations

$$
\dot{x}=a(x), \dot{y}=b(x)+c(x) y+d(x) y^{2}
$$

In [8] (Lemma 5) the following result was proved.

Theorem [8]. If the equation (5) has an invariant algebraic curve $K(x, y)=0$ which does not contain any line $x=$ const, then we have one of the following four possibilities.

(a) There is only one invariant algebraic curve, a rational curve $y=R(x)$, where $R$ is a rational function, and the system has a first integral of the DarbouxSchwartz-Christoffel type

$$
H=(y-R(x))^{-1} e^{g(x)} \prod\left(x-x_{i}\right)^{a_{i}}+\int^{x} e^{g(s)} \prod\left(s-x_{i}\right)^{a_{i}-1} e^{g(s)} P(s) d s,
$$

( $P$ is a polynomial, $g$ is a rational function, and $x_{i}, a_{i} \in \mathbb{C}$ ).

(b) There are exactly two rational invariant curves $y=R_{1,2}(x)$, and the system has a first integral of the Darboux type

$$
H=\frac{y-R_{1}(x)}{y-R_{2}(x)} e^{g(x)} \prod\left(x-x_{i}\right)^{a_{i}} .
$$


(c) The curve is hyperelliptic $(y+P(x))^{2}=Q(x)$ ( $P$ and $Q$ are rational functions), and the system has a first integral of the Darboux-hyperelliptic type

$$
H=\frac{y+P(x)+\sqrt{Q(x)}}{y+P(x)-\sqrt{Q(x)}} \exp \left[\int^{x} W(s) \sqrt{Q(s)} d s\right],
$$

( $W$ is a rational function)

(d) The curve is $d$-fold, $d>2$, and the system (5) has a rational first integral.

Remark. The Liénard system with rational invariant curve or hyperelliptic invariant curve can be non-integrable in quadratures. The elementary first integrals for this system were studied by Odani in [4].

In the last section we discuss Yablonsky's example of an algebraic limit cycle for a quadratic system.

The next four sections are devoted to the proof of Theorems 1 and 2 .

\section{EXAMPLES OF SYSTEMS WITH INVARIANT ALGEBRAIC CURVES AND ALGEBRAIC LIMIT CYCLES}

2.1. A rational curve. (Here and in what follows the lower index of a polynomial always denotes its degree). We assume that the curve is of the form

$$
y=P(x),
$$

where $P(x)$ is a polynomial. It is invariant for (1) iff

$$
g_{n}=-\left(f_{m}+P^{\prime}\right) P \text {. }
$$

If $m<n \leq 2 m+1$ then we can choose $P$ in one of the following two forms: either

(i) $P(x)=P_{n-m}(x)$ of degree $n-m$, or

(ii) $P(x)=P_{m+1}(x)=-F(x)+Q_{n-m}(x)$ with $F^{\prime}=f$ and $Q_{n-m}$ of degree $n-m$.

If $n=2 k-1>2 m+1$, then we choose $P=P_{k}$ of degree $k$.

The above gives the proof of the part (a) of Theorem 1 in all cases except for even $n=2 k>2 m+1$.

Remarks. If $n$ is even and $n>2 m+1$, then there cannot be such an invariant curve and the above cases are the only ones with 1 -fold invariant curves (see the next section).

One can easily check that such curves cannot exist in Odani's case $n \leq m$.

A rational curve of the above form cannot be a limit cycle.

\subsection{A hyperelliptic curve. We assume that}

$$
K(x, y)=(y+P(x))^{2}-Q(x)=0,
$$

where $P=P_{m+1}$ is a polynomial of degree $m+1$ and $Q$ is also a polynomial. The condition of invariance of the curve $K=0$, i.e. $\left.\dot{K}\right|_{K=0}=0$, leads to

$$
\begin{gathered}
f_{m}=P^{\prime}+\frac{1}{2} P \frac{Q^{\prime}}{Q}, \\
g_{n}=-\frac{1}{2}\left(Q^{\prime}-P^{2} \frac{Q^{\prime}}{Q}\right) .
\end{gathered}
$$


Let

$$
P=a \prod_{1}^{r}\left(x-x_{i}\right)^{m_{i}}, \sum m_{i}=m+1, x_{i} \neq x_{j} .
$$

In order to compensate for the poles in $Q^{\prime} / Q$ we must have

$$
Q=b \prod_{1}^{r}\left(x-x_{i}\right)^{n_{i}} .
$$

Note that the polynomial $P^{\prime}+\frac{1}{2} P \frac{Q^{\prime}}{Q}=P \sum \frac{m_{i}+n_{i} / 2}{x-x_{i}}=a M x^{m}+\ldots, M>0$, is of degree $\operatorname{deg} P-1=m$. This shows that our choice of the degree of $P$ is the only possible one. Let us look at the degree of $Q$.

If $n>2 m+1$, then we choose $Q=Q_{n+1}$ of degree $n+1$ with the sole restriction (9). Then $g_{n}$ will be of degree $n$.

At this moment we complete the proof of the part (a) of Theorem 1 (in the case $n=2 k>2 m+1)$.

If $n=2 m+1$, then we have the restriction $\operatorname{deg}\left(P^{2}-Q\right)=2 m+2$. Thus $\operatorname{deg} Q \leq 2 m+2$. Notice that in the example (3) one has $m=2, n=5$ but $\operatorname{deg} Q=2$.

If $n<2 m+1$, then in order to get $\operatorname{deg} g_{n}=n$ we must have

$$
\operatorname{deg}\left(P^{2}-Q\right)=n+1 .
$$

The latter condition means that $\operatorname{deg} Q=2 m+2, b=a^{2}$ in (9) and the coefficients preceeding $x^{i}, i=n+1, \ldots, 2 m+1$, of the polynomial $P^{2}-Q$ vanish. This implies that $P^{2} / Q=1+O\left(x^{n-2 m-1}\right)$ or that $\ln \left(P^{2} / Q\right)=\sum\left(2 m_{i}-n_{i}\right) \ln \left(x-x_{i}\right)=$ $O\left(x^{n-2 m-1}\right)$. Therefore

$$
\begin{gathered}
\sum\left(2 m_{i}-n_{i}\right) x_{i}^{0}=0, \\
\sum\left(2 m_{i}-n_{i}\right) x_{i}^{1}=0, \\
\ldots\left(2 m_{i}-n_{i}\right) x_{i}^{2 m-n}=0 .
\end{gathered}
$$

We have $2 m-n$ equations in $r$ variables $x_{i}, x_{i} \neq x_{j}$ (the first equation is trivial). If $r \leq 2 m-n+1$, then from the Vandermonde formula we obtain that $2 m_{i}=n_{i}, i=1, \ldots, r$. So $Q=P^{2}$ and $g_{n} \equiv 0$, in contradiction with the assumption (2).

Thus $r>2 m-n+1$. Since $r \leq m+1$, we get the condition $m<n$. We see that in Odani's case there cannot be a hyperelliptic invariant algebraic curve.

2.3. Hyperelliptic limit cycles. Now we look at the possibility of the existence of a limit cycle in the form of a real oval of a hyperelliptic curve (6). Observe that if a limit cycle exists, then the coefficients of the polynomials $P$ and $Q$ must be real. Because a limit cycle is smooth, after some renumeration of the roots $x_{i}$ we must have

$$
Q(x)=b\left(x-x_{1}\right)\left(x-x_{2}\right) \prod_{2}^{r}\left(x-x_{i}\right)^{n_{i}}
$$

and $Q(x)>0$ for $x_{1}<x<x_{2}$. Moreover, we can assume that $x_{1}=0, x_{2}=1$. We seek a limit cycle $\gamma$ as an oval of the curve (6) situated in the strip $0<x<1, y \in \mathbb{R}$. Moreover, we see that $m>0$. 
(Note that if $m=0$ and $V$ denotes the vector field (1), then $\operatorname{div} V=-f_{0}=$ const $\neq$ 0 , which also gives nonexistence of limit cycles.)

If $m=1$, then $P=a x(x-1), Q=b x(x-1), b<0$ and $n=3$. But then one can take the variables $X=x(x-1), Y=y$, in which the system (1) becomes linear with the first integral $(Y-c X)^{\alpha}(Y-c X)^{\beta}$. Thus if $m=1$, then $K=0$ cannot contain a limit cycle.

Let $m>1$. A limit cycle $\gamma$ surrounds a real singular point $x=x_{0}, y=$ $0, g_{n}\left(x_{0}\right)=0$ of the anti-saddle type (at least one). We have $\operatorname{Tr} d V\left(x_{0}, 0\right)=$ $-f_{m}\left(x_{0}\right)$, which must be non-zero (i. e. $\left(x_{0}, 0\right)$ is a focus or a node) if $\gamma$ is a limit cycle. By (7) and (8), we find the following condition:

$$
\left[\left(P^{2}-Q\right) Q^{\prime}\right]\left(x_{0}\right)=0,\left(P^{2} Q\right)^{\prime}\left(x_{0}\right) \neq 0 .
$$

If $P$ and $Q$ depend on parameters $\delta_{1}, \ldots, \delta_{s}$, then $x_{0}=x_{0}\left(\delta_{1}, \ldots, \delta_{s}\right)$, and we need the property $\left(P^{2} Q\right)^{\prime}\left(x_{0}\left(\delta_{1}, \ldots, \delta_{s}\right)\right) \not \equiv 0$.

If $n \geq 2 m+1, m>1$, then the above condition is easily achieved $(r=m+1, s=$ $m-1)$.

If $m=2, n<5$, then $P(x)=a x(x-1)\left(x-x_{3}\right), Q(x)=a^{2} x(x-1)\left(x-x_{3}\right)^{4}$, and we see that the condition $Q(x)>0$ for $0<x<1$ does not hold.

Assume that $3 \leq m<n<2 m+1$. Because we want to have as much freedom as possible, we take the number of variables $x_{i}$ to be maximal; so $P(x)=$ $\left(x-x_{1}\right) \ldots\left(x-x_{m+1}\right)\left(m_{1}=\ldots=m_{m+1}=1\right)$. Also we take the polynomial $Q$ in the form

$$
Q(x)=\left(x-x_{1}\right) \ldots\left(x-x_{k}\right)\left(x-x_{k+1}\right)^{n_{k+1}} \ldots\left(x-x_{m+1}\right)^{n_{m+1}},
$$

where $n_{j} \geq 3$ for $j \geq n+1$. If some $n_{j}=2$, then the variable $x_{j}$ does not appear in the equations (10) and we have less freedom in solving them. For fixed $k, n_{k+1}, \ldots, n_{m+1}$, we denote $y_{1}=x_{k+1}, k_{1}=n_{k+1}-2, \ldots, y_{s}=x_{m+1}, k_{s}=$ $n_{m+1}-2, s=m-k+1$. Then, the system (10) takes the form

$$
\sum_{i-1}^{k} x_{i}^{j}=\sum_{i=1}^{s} k_{i} y_{i}^{j}, j=1, \ldots, 2 m-n
$$

$\left(\sum k_{i}=k\right)$

Our goal is to find a limit cycle for $n>m+1$. It suffices to find a limit cycle for $n=m+2$ because, when $n>m+2$, then we have fewer equations in (10), and after perturbation of the solution obtained for $n=m+2$ we get a limit cycle (the structural stability).

2.4. Lemma. If $m>2, n=m+2$, then there is a system from $\mathcal{A}_{m, n}$ with a limit cycle in the curve $K(x, y)=0$.

Proof. We fix $k=m-1, k_{1}=1, k_{2}=m-2$ and consider the system (12). One can see that the system

$$
\begin{aligned}
& x_{1}+\ldots+x_{m-1}=y_{1}+(m-2) y_{2}, \\
& x_{1}^{m-2}+\ldots+x_{m-1}^{m-2}=y_{1}^{m-2}+(m-2) y_{2}^{m-2}
\end{aligned}
$$

has the solution $x_{1}(0)=\ldots=x_{m-2}(0)=y_{2}(0)=0, x_{m-1}(0)=y_{1}(0)=1$. We shall perturb it. We choose $x_{i}(t)=\lambda_{i} t+\ldots, i=1, \ldots, m-2, y_{2}(t) \equiv 0, y_{1}(t) \equiv$ 
$1, x_{m-1}(t)=1-t^{m-2}+\ldots$ (the positions of two points are fixed). We obtain the system of equations

$$
\begin{gathered}
\left(\lambda_{1}+\ldots \lambda_{m-2}\right) t=\ldots, \\
\left(\lambda_{1}^{2}+\ldots \lambda_{m-2}^{2}\right) t^{2}=\ldots, \\
\ldots \ldots \ldots \ldots \ldots \ldots \ldots \ldots \ldots \ldots \ldots \ldots \\
\left(\lambda_{1}^{m-2}+\ldots \lambda_{m-2}^{m-2}\right) t^{m-2}=t^{m-2}+\ldots
\end{gathered}
$$

So, we choose $\lambda_{j}=e^{2 \pi i j /(m-2)}$ and we get

$$
\begin{aligned}
& P_{t}(x) \approx\left(x^{m-2}-t^{m-2}\right)\left(x-1+t^{m-2}\right)(x-1) x, \\
& Q_{t}(x) \approx\left(x^{m-2}-t^{m-2}\right)\left(x-1+t^{m-2}\right)(x-1)^{3} x^{m} .
\end{aligned}
$$

We see that for $t>0$ there are exactly two simple zeroes of $Q_{t}(x)$ between 0 and 1 : $x_{m-2}(t)=t+\ldots$ and $x_{m-1}(t)=1-t^{m-2}+\ldots$ Next, $Q_{0}(x)=(x-1)^{4} x^{2 m-2}>0$ for $0<x<1$ and hence $Q_{t}(x)>0$ for $x_{m-2}(t)<x<x_{m-1}(t)$; there is a real oval $\gamma$ situated in the strip $x_{m-2}(t)<x<x_{m-1}(t)$. We claim that it is a limit cycle.

We need to check that inside $\gamma$ there is only one singular point for which the conditions (11) hold. We have

$$
\begin{aligned}
& P_{t}^{2}-Q_{t} \approx t^{m-2}\left(x^{m-2}+1-x\right)(x-1)^{3} x^{m}=t^{m-2} S(x), S>0, \\
Q_{0}^{\prime}(x)= & \left.2[(m+1) x-(m-1)] R, R>0 \text { (hence } x_{0} \approx(m-1) /(m+1)\right), \text { and } \\
S^{\prime}\left(x_{0}\right)= & \left(x_{0}-1\right)^{2} x_{0}^{m-1}\left[x_{0}^{m-2}\left((2 m+1) x_{0}-2 m+2\right)+\left(1-x_{0}\right)\left((m+4) x_{0}-m\right)\right] \\
= & \frac{\left(x_{0}-1\right)^{2} x_{0}^{m-1}}{(m+1)^{m-1}}\left[(m-1)^{m-1}-4(m-2)(m+1)^{m-3}\right] .
\end{aligned}
$$

If $S^{\prime}\left(x_{0}\right)=0$, then $m$ is odd, $m=2 l+1$ and $l^{2 l}=(2 l-1)(l+1)^{2 l-2}$. The only solution of the last equation is $l=1$ (consider the cases of even and odd $l$ ). So for $m>3$ the point $\left(x_{0}, 0\right)$ is the unique singular point inside $\gamma$; it is not a center and $\gamma$ is a limit cycle.

If $m=3, n=5$, then the above choice of the polynomials $P$ and $Q$ does not give limit cycles. Instead we choose $P(x)=x(x-1)(x+1-3 t)(x-t), Q(x)=$ $x(x-1)(x+1-3 t)(x-t)^{5}$. We have $P^{2}-Q=P(x)(x-t)\left[x(x-1)(x+1-3 t)-(x-t)^{3}\right]$ (of degree $6=n+1$ ). If $t \rightarrow \infty$, then $\left(P^{2}-Q\right)(x) \neq 0$ for $0<x<1$ and $Q^{\prime}(x)=$ $3(2 x-1) t^{6}+O\left(t^{5}\right)$. Therefore there is a unique singular point $\left(x_{0}, 0\right), x_{0} \approx 1 / 2$, of the system (1) inside the oval $\gamma$. Moreover, $(Q / P)^{\prime}\left(x_{0}\right)=4\left(x_{0}-t\right) \neq 0$. This shows that $\gamma$ is a limit cycle.

2.5. Lemma. (a) If $n=m+1, k=m$, then all solutions of the system (12) are equivalent to $P(x)=x\left(x^{m}-d\right), Q(x)=x^{m+2}\left(x^{m}-d\right)$. Moreover, the hyperelliptic curve is not a limit cycle.

(b) Otherwise the only solutions of the system (12) are such that $Q(x)=P^{2}(x)$. Moreover, the corresponding algebraic trajectories are not limit cycles. 
The possibility of the existence of hyperelliptic limit cycles is presented in the following table:

$$
\begin{array}{cc}
m=0 & N O \\
m=1 & N O \\
m=2, n<5 & N O \\
m=2, n \geq 5 & Y E S \\
m \geq 3, n \leq m+1 & N O \\
m \geq 3, n>m+1 & Y E S
\end{array}
$$

This shows point (c) of Theorem 1 and its point (d) restricted to the case of hyperelliptic limit cycles.

Proof of Lemma 2.5. (a) We have $s=1$, and we can assume that $y_{1}=0$. Then, we obtain the system $x_{1}+\ldots+x_{m}=0, \ldots, x_{1}^{m-1}+\ldots+x_{m}^{m-1}=0$. So, the polynomial $R(\lambda)=\prod\left(\lambda-x_{i}\right)$ is of the form $R(\lambda)=\lambda^{m}-d$.

(b) The system (12) has $k ! /\left(k_{1} ! \ldots k_{s} !\right)$ solutions of the form

$$
x_{\sigma(1)}=x_{\sigma(2)}=\ldots=x_{\sigma\left(k_{1}\right)}=y_{1}, x_{\sigma\left(k_{1}+1\right)}=\ldots=x_{\sigma\left(k_{1}+k_{2}\right)}=y_{2}, \ldots, x_{k}=y_{s}
$$

of codimension $k$ and degree 1 , where $\sigma$ are permutations of the set $\{1, \ldots, k\}$. We claim that these are all of the solutions of the system (12) in $\mathbb{C} P^{k+s-1}$ for $y_{i} \neq y_{j}$.

To show this we use algebraic intersection theory. We treat these equations as equations for $x_{i}$ with fixed $y_{j}, y_{i} \neq y_{j}$, and we show that the system of the first $k$ of the equations (12) has the above set of solutions. The degree of the algebraic variety defined by them is $k$ !. If we show that the intersection index at each of the given solutions is $k_{1} ! \ldots k_{s} !$, then we shall get the result. It is clear that this index does not depend on the particular solution.

We put $x_{k_{i}+j}=y_{i}+\lambda_{i j}$. We have to calculate the index at $\lambda=0$ of the map

$$
\lambda \rightarrow\left(f_{1}(\lambda), \ldots, f_{k}(\lambda)\right), f_{r}(\lambda)=\sum_{i, j}\left[\left(y_{i}+\lambda_{i j}\right)^{r}-y_{i}^{r}\right] .
$$

2.6. Lemma. There is an invertible linear transformation

$$
\left(f_{1}, \ldots, f_{k}\right) \rightarrow\left(g_{11}, \ldots, g_{1 k_{1}}, g_{21}, \ldots, g_{s k_{s}}\right)
$$

such that

$$
g_{i l}=\mu_{i l}+g_{i l}^{\prime}, \mu_{i l}=\sum_{j=1}^{k_{i}} \lambda_{i j}^{l}
$$

and the $g_{i l}^{\prime}$ are of higher order with respect to $\lambda$.

From this lemma we get that the intersection index of the map $\lambda \rightarrow f(\lambda)$ is the same as the intersection index of the map $\lambda \rightarrow\left(\mu_{i l}\right)$, which equals $k_{1} ! \ldots k_{s}$ !

Proof. We have

$$
f_{r}(\lambda)=\sum_{i} \sum_{l}\left(\begin{array}{l}
r \\
l
\end{array}\right) y_{i}^{r-l}\left(\sum_{j} \lambda_{i j}^{l}\right)=\sum_{i, l}\left(\begin{array}{l}
r \\
l
\end{array}\right) y_{i}^{r-l} \mu_{i l} .
$$


The matrix of the transformation $(\mu) \rightarrow f$ is of the form

$$
\left[\begin{array}{cccccccc}
1 & 0 & 0 & \ldots & 1 & 0 & 0 & \ldots \\
\left(\begin{array}{l}
2 \\
1
\end{array}\right) y_{1} & 1 & 0 & \ldots & \left(\begin{array}{l}
2 \\
1
\end{array}\right) y_{2} & 1 & 0 & \ldots \\
\left(\begin{array}{l}
3 \\
1
\end{array}\right) y_{1}^{2} & \left(\begin{array}{l}
3 \\
2
\end{array}\right) y_{1} & 1 & \ldots & \left(\begin{array}{l}
3 \\
1
\end{array}\right) y_{2}^{2} & \left(\begin{array}{l}
3 \\
2
\end{array}\right) y_{2} & 1 & \ldots \\
\left(\begin{array}{l}
4 \\
1
\end{array}\right) y_{1}^{3} & \left(\begin{array}{l}
4 \\
2
\end{array}\right) y_{1}^{2} & \left(\begin{array}{l}
4 \\
3
\end{array}\right) y_{1} & \ldots & \left(\begin{array}{l}
4 \\
1
\end{array}\right) y_{2}^{3} & \left(\begin{array}{l}
4 \\
2
\end{array}\right) y_{2}^{2} & \left(\begin{array}{l}
4 \\
3
\end{array}\right) y_{2} & \ldots \\
\ldots & \ldots & \ldots & \ldots & \ldots & \ldots & \ldots & \ldots
\end{array}\right] .
$$

We have to show that this matrix is invertible.

Introduce the auxiliary functions

$$
\begin{gathered}
h_{r 1}(\nu ; y)=(y+\nu)^{r}-y^{r} \\
h_{r 2}(\nu ; y)=(y+2 \nu)^{r}-\left(\begin{array}{l}
2 \\
1
\end{array}\right)(y+\nu)^{r}+y^{r}, \\
\ldots \ldots \ldots \ldots \ldots \ldots \ldots \ldots \ldots \ldots \ldots \ldots \ldots \ldots . . \\
h_{r t}(\nu ; y)=(y+t \nu)^{r}-\left(\begin{array}{l}
t \\
1
\end{array}\right)(y+(t-1) \nu)^{r}+\ldots+(-1)^{t} y^{r} .
\end{gathered}
$$

We have $h_{r t}(\nu ; y)=\left.\left(1-e^{-\partial / \partial x}\right)^{t}(y+x \nu)^{r}\right|_{x=t}=\left[\left(\begin{array}{l}r \\ t\end{array}\right) y^{r-t}\right] t ! \nu^{t}+O\left(\nu^{t+1}\right)$ as $\nu \rightarrow 0$ $(t \leq r)$

Therefore the matrix (13) has the same entries as the leading coefficients before $t ! \nu^{t}$ of the functions $h_{r t}\left(\nu ; y_{i}\right), i=1, \ldots, s, r=1, \ldots, k_{i}, t=1, \ldots, r$. But the functions $h_{r t}\left(\nu ; y_{i}\right)$ are expressed in terms of the functions $\left(y_{i}+j \nu\right)^{r}$, power functions at $k$ different points. The determinant of the matrix

$$
\left[\begin{array}{ccccc}
\left(y_{1}+\nu\right)-y_{1} & \left(y_{1}+2 \nu\right)-\left(y_{1}+\nu\right) & \ldots & \left(y_{2}+\nu\right)-y_{2} & \ldots \\
\left(y_{1}+\nu\right)^{2}-y_{1}^{2} & \left(y_{1}+2 \nu\right)^{2}-\left(y_{1}-\nu\right)^{2} & \ldots & \left(y_{2}+\nu\right)^{2}-y_{2}^{2} & \ldots \\
\ldots & \ldots & \ldots & \ldots & \ldots
\end{array}\right]
$$

is nonzero. From this we get that the determinants of the matrices

$$
\left[\begin{array}{cccccc}
h_{11}\left(y_{1} ; \nu\right) & 0 & \ldots & h_{11}\left(y_{2} ; \nu\right) & 0 & \ldots \\
h_{21}\left(y_{1} ; \nu\right) & h_{22}\left(y_{1} ; \nu\right) & \ldots & h_{21}\left(y_{2} ; \nu\right) & h_{22}\left(y_{2} ; \nu\right) & \ldots \\
\ldots & \ldots & \ldots & \ldots & \ldots & \ldots
\end{array}\right]
$$

as $\nu \rightarrow 0$ and (13) are nonzero too.

2.7. $d$-fold curves, $d>2$. We consider the situation when $n=2 m+1$ and

$$
g_{n}=\Lambda f_{m} F,
$$

where $F(x)$ is a polynomial such that $F^{\prime}=f_{m}$ and $\Lambda$ is a constant. (One can say that the primitive of $g_{n}$ is proportional to $F^{2}$.) Then the vector field (1) can be pushed forward by means of the map $(x, y) \rightarrow(X, Y), X=F(x), Y=y$. Indeed after transformation and division by $f_{m}$ we obtain the linear system

$$
\dot{X}=Y, \dot{Y}=-\Lambda X-Y \text {. }
$$

If $\nu_{1,2}$ are its eigenvalues, then the system has a first integral of the Darboux type

$$
H=(Y-c X)^{\nu_{1}}(Y-d X)^{-\nu_{2}}
$$

( $c$ and $d$ are constants). If $\lambda=\nu_{1} / \nu_{2}$ is a rational number, then the first integral can be chosen as a rational function, and any phase curve $H=$ const is algebraic.

Of course, we cannot obtain a limit cycle in this way. 
Another example of a $d$-fold, $d \geq 3$, invariant curve provides the Wilson system (3). (I owe this remark to Odani.) It has three invariant curves:

$$
\Phi_{1,2}=y+\frac{\mu}{4} x\left(x^{2}-2\right) \pm \frac{\lambda}{2} x=0, \quad \lambda=\sqrt{\mu^{2}-4},
$$

and

$$
\Phi_{3}=\left[y+\frac{\mu}{4} x\left(x^{2}-4\right)\right]^{2}+x^{2}-4=0
$$

(see the Introduction). So, the curve $\Phi_{1} \Phi_{2} \Phi_{3}=0$ is 4 -fold. Odani [4] has shown that the Wilson system has non-rational Darboux type first integral

$$
H=\Phi_{3} \Phi_{1}^{\mu / \lambda-1} \Phi_{2}^{-\mu / \lambda-1} .
$$

It would be interesting to know whether there can exist a $d$-fold curve, $d \geq 3$, with a limit cycle, whether a system with such a curve is always integrable in quadratures, and whether irreducible such curves can exist.

\section{THE BEHAVIOUR OF TRAJECTORIES NEAR INFINITY}

After proving the existence results we pass to the classification of invariant algebraic curves of Liénard systems. The natural beginning of such a study is to look at the asymptotic behaviour of these curves. In this section we investigate the analytic properties of individual trajectories. After a suitable blowing-up of a singular point at infinity we obtain a resolved vector field with some new singular points. We apply the theory of normal forms to study phase curves near each of these points. In the next section we will study trajectories near a whole invariant divisor containing these points.

3.1. Lemma. If $m<n<2 m+1$, then the asymptotic behaviours of trajectories tending to infinity for $V \in \mathcal{A}_{m, n}$ are the following:

(i) The trajectories $\Gamma_{\mu}^{0}, \mu \in \mathbb{C}$ :

$$
y=c_{n-m}^{(0)} x^{n-m}+c_{n-m-1}^{(0)}+\ldots+\mu \cdot O\left(e^{-a x^{l}}\right), x \rightarrow \infty,
$$

with uniquely defined constants $c_{i}^{(0)}, i=\ldots, n-m$. Only one of these curves, $\Gamma_{0}^{0}$, can be analytic (but it is possible that none of them are analytic at infinity).

(ii) the 1-parameter family of trajectories

$$
\Gamma_{\mu}: y=c_{m+1} x^{m+1}+\ldots+c_{1} x+\mu+d \ln x+c_{-1} x^{-1}+\ldots, \mu \in \mathbb{C},
$$

where $c_{m+1}, \ldots, c_{1}, d$ are defined uniquely (as polynomials of $a_{i}, b_{j}$ ). If $d=0$ then then $\Gamma_{\mu}$ are analytic near infinity.

Remark. The thesis of this lemma holds also in the Odani's case $m \geq n$.

3.2. Lemma. (a) If $2 m+1<n$ and $n$ is even, then there is the 1-parameter family of trajectories $\Delta_{\mu}$ :

$$
y^{2}=c_{n+1} x^{n+1}+\ldots+c_{1} x+\left(d_{m_{1}} x^{m+1}+\ldots\right) y+\mu+d \ln x+\ldots, \mu \in \mathbb{C},
$$

where $c_{n+1}, \ldots, c_{1}, d_{m+1}, \ldots, d$ are defined uniquely. If $d=0$, then $\Delta_{\mu}$ are analytic near infinity.

(b) If $2 m+1<n=2 k-1$, then there are the two 1-parameter families of trajectories

$$
\Delta_{\mu}^{ \pm}: y=c_{k}^{ \pm} x^{k}+\ldots+c_{1-k}^{ \pm} x^{1-k}+\mu x^{-k}+d^{ \pm} x^{-k} \ln x+\ldots, \mu \in \mathbb{C},
$$


where $c_{k}^{ \pm}, \ldots, c_{1-k}^{ \pm}, d^{ \pm}$are defined uniquely and for $d^{ \pm}=0$ these curves are analytic near infinity.

3.3. Lemma. If $n=2 m+1$, then we consider the roots $\lambda_{1,2}$ of the equation (4) (see Theorem 2).

(a) If $\lambda_{1}, \lambda_{2} \notin \mathbb{Q}$, then the system (1) has exactly two analytic trajectories near infinity:

$$
\Xi_{1,2}: y=\eta_{1,2}(x), \eta_{1,2}=c_{m+1}^{(1,2)} x^{m+1}+c_{m}^{(1,2)} x^{m}+\ldots
$$

where $c_{m+1}^{(1,2)}=a_{m} \lambda_{1,2} /\left(1-2(m+1) \lambda_{1,2}\right)$.

(b) If $\lambda_{i}=p_{i} / q_{i} \in \mathbb{Q}, i=1,2, \lambda_{1,2}>0$ and $\lambda_{1,2} \neq 1 / N$ with $N$ an integer, then there are the two families of analytic trajectories

$$
\Xi_{i, \mu}:\left(y-\eta_{i}(x)\right)^{q_{i}}=\mu x^{(m+1) q_{i}-p_{i}}(1+\ldots), \mu \in \mathbb{C}, i=1,2 .
$$

(c) If $\lambda_{1}<0<\lambda_{2} \in \mathbb{Q}, \lambda_{2} \neq 1 / N, N$ an integer and $\lambda_{1} / \lambda_{2}=-p / q$, then there is the family of analytic curves

$$
\Xi_{2, \mu}:\left(y-\eta_{2}(x)\right)^{q}=\mu\left(y-\eta_{1}(x)\right)^{p}(1+\ldots), \mu \in \mathbb{C},
$$

and the analytic curve $\Xi_{1}$

(d) If $\lambda_{2}=1 / N$, where $N>0$ is an integer, then there is the family of trajectories $\Xi_{2, \mu}$ :

$$
y=c_{m+1}^{(2)} x^{m+1}+\ldots+c_{m-N+2}^{(2)} x^{m-N+2}+\mu x^{m-N+1}+d x^{m-N+1} \ln x+\ldots,
$$

$\mu \in \mathbb{C}$, where $c_{j}^{(2)}$ and $d$ are defined uniquely. If $d=0$, then these trajectories are analytic and we have the situation as in (b) or (c).

3.4. The first terms of the asymptotics. If $n>m+1$, then the system (1) has only one singular point at infinity $(0: 1: 0)$ (in $\left.\mathbb{C} P^{2}=\{(x: y: z)\}\right)$. If $n=m+1$, then besides $(0: 1: 0)$ there is also the point $\left(a_{m}:-b_{m+1}: 0\right)$.

Near $(0: 1: 0)$ there are the following possible behaviours of an eventual algebraic phase curve: either

(i) $x=x_{0}+C y^{-\alpha}+\ldots, \alpha>0$ as $y \rightarrow \infty$ or

(ii) $y \sim C x^{\alpha}, \alpha>1$, as $x \rightarrow \infty$.

But in the case (i) we have $d x / d y \sim y^{-\alpha-1} \rightarrow 0$ on the one hand and $d x / d y=$ $-\left(f_{m}(x)+g_{n}(x) / y\right)^{-1} \sim f_{m}^{-1}\left(x_{0}\right) \nrightarrow 0$ on the other.

In case (ii) we have

$$
C \alpha x^{\alpha-1} \sim-a_{m} x^{m}-\left(b_{n} / C\right) x^{n-\alpha} .
$$

Now we have three possibilities:

(i) If the term $a_{m} x^{m}$ is dominating in the right hand side, then $\alpha=m+1$ and $m>n-\alpha($ or $n<2 m+1)$.

(ii) If the term $\left(b_{m} / C\right) x^{n-\alpha}$ is dominating, then $\alpha=(n+1) / 2$ and $n-\alpha>m$ (or $n>2 m+1)$.

(iii) If both terms are of equal order, then $\alpha=n-m$ and $\alpha-1 \leq m$, which gives $n \leq 2 m+1$. Here we can also include the case $\alpha=1$ for $n=m+1$.

In the next step of our analysis we perform a blowing-up of the singular point $(0: 1: 0)$ and use the the local theory of analytic vector fields.

\subsection{Analytic orbital normal form theorem. Let}

$$
\dot{x}=\nu_{1} x+\ldots, \dot{y}=\nu_{2} y+\ldots
$$


be a germ of an analytic vector field in $\mathbb{C}^{2}$ near the isolated elementary (i.e. $\left|\nu_{1}\right|+$ $\left.\left|\nu_{2}\right| \neq 0\right)$ singular point $(0,0)$.

(a) If $\nu_{1} \nu_{2} \neq 0$ and $\lambda=\nu_{1} / \nu_{2} \notin \mathbb{Q}$ (non-resonant point), then exactly two analytic invariant curves (separatrices) pass through the point $(0,0)$. The system is formally equivalent to its linear part $\dot{\tilde{x}}=\nu_{1} \tilde{x}, \dot{\tilde{y}}=\nu_{2} \tilde{y}$ with the first integral

$$
H=\tilde{x} \tilde{y}^{-\lambda} \text {. }
$$

(b) If $\nu_{1}=0 \neq \nu_{2}$ (semi-hyperbolic point) then there are two distinguished invariant trajectories: an analytic separatrix corresponding to the eigenvalue $\nu_{2}$ and a center manifold which may be not analytic and may be not unique. If the center manifold is analytic, then it is unique (others are not analytic). The formal orbital normal form is $\dot{\tilde{x}}=\tilde{x}^{l+1} / l, \dot{\tilde{y}}=\tilde{y}\left(1+b \tilde{x}^{l}\right)$ with the first integral

$$
H=\tilde{y} \tilde{x}^{b l} e^{-1 / \tilde{x}^{l}}
$$

(c) If $\lambda=N$ is a positive integer ( $N: 1$ resonant node), then the system is analytically equivalent to the system

$$
\dot{\tilde{x}}=N \tilde{x}+\delta \tilde{y}^{N}, \dot{\tilde{y}}=\tilde{y}
$$

with the 1-parameter family of trajectories

$$
\tilde{x} \tilde{y}^{-N}-\delta \ln \tilde{y}=\mu, \quad \mu \in \mathbb{C}
$$

(or $H=\tilde{y}^{\delta} e^{-\tilde{x} / \tilde{y}^{N}}=$ const), which are analytic for $\delta=0$. If $\delta \neq 0$, then only the curve $\tilde{y}=0$ is analytic.

(d) If $\lambda=p / q>1$ is rational but not an integer ( $p: q$ resonant node), then the system is analytically equivalent to $\dot{\tilde{x}}=p \tilde{x}, \dot{\tilde{y}}=q \tilde{y}$ with the 1-parameter family of analytic trajectories

$$
H=\tilde{x}^{q} \tilde{y}^{-p}=\mu, \quad \mu \in \mathbb{C} P^{1} .
$$

(e) If $\lambda=-p / q$ is negative and rational ( $p:-q$ resonant saddle), then the system has two analytic invariant curves passing through $(0,0)$. It is formally orbitally equivalent to $\dot{\tilde{x}}=\tilde{x}\left(p+u^{l} /\left(1+b u^{l}\right)\right), \dot{\tilde{y}}=-q \tilde{y}, u=\tilde{x}^{q} \tilde{y}^{p}$ with the first integral

$$
H=\tilde{x}^{A} \tilde{y}^{B} e^{-1 / u^{l}} \text {. }
$$

If $l=\infty$, then the system is analytically linearizable with the first integral $u$.

This theorem contains a significant part of the local theory of analytic planar vector fields. Its proof or references to them can be found in [1].

Remark. We note that there are some situations when in the non-resonant case the normalizing transformation is analytic: $\lambda \in \mathbb{C} \backslash \mathbb{R}$ or $\lambda<0$ is weakly approximated by rationals. In the latter case $\lambda$ satisfies the so-called Bruno condition (with geometric interpretation given by Yoccoz).

If $\lambda=0$ or $\lambda=-p / q$, then the transformation leading to the normal form is analytic in some sectorial domains. The analytic normal form contains the so-called Martinet-Ramis-Ecalle-Voronin functional moduli, the "differences" between normalizing transformations in different sectorial domains.

3.6. Proof of Lemma 3.1. If $m<n<2 m+1$, then we introduce the variables

$$
z=1 / x, u=y / x^{m+1} \text {. }
$$


We get

$$
\dot{z}=z u, \dot{u}=a_{m} u+(m+1) u^{2}+a_{m-1} z u+\ldots+\left(a_{n-m-1} u+b_{n}\right) z^{2 m+1-n}+\ldots .
$$

This system has two singular points: $z=0, u=u_{0}=-a_{m} /(m+1)$ and $z=$ $0, u=0$.

The point $(0,0)$ is semi-hyperbolic with eigenvalues 0 and $a_{m} \neq 0$, and the integer $l=n-2 m-1$. From Theorem 3.5 it follows that it has a center manifold $\tilde{y}=u+\left(b_{n} / a_{m}\right) z^{n-2 m-1}+\ldots=0$ with uniquely defined Taylor series. In the old coordinates it is of the form $y=-g_{n}(x) / f_{m}(x)+O\left(x^{2 n-3 m-1}\right)$. If it is analytic then it is unique. The trajectories $\Gamma_{\mu}^{0}$ are given by $\tilde{y} \tilde{x}^{b l} e^{-1 / \tilde{x}^{l}}=\mu$.

The point $\left(0, u_{0}\right)$ is an $(m+1): 1$ resonant node with the normal form (14) and the family of trajectories $\Gamma_{\mu}$ (15) which are analytic for $\delta=0$. The parameter $\delta$ from Theorem 3.5 (c) corresponds to $d$ in Lemma 3.1.

The Taylor expansions of the curves $\Gamma$ and $\Gamma_{\mu}$ can be obtained by comparision of the coefficients in the equation $d y / d x=-f_{m}(x)-g_{n}(x) / y$. This remark remains valid in the proofs of the other two lemmas.

3.7. Proof of Lemma 3.2. (a) If $n=2 k>2 m+1$, then we apply the following blowing-up:

$$
z=y / x^{k+1}, u=y^{2} / x^{2 k+1}
$$

(we have $x=u / z^{2}, y=u^{k+1} / z^{2 k+1}$ ) to get

$$
\dot{z}=z\left[b_{2 k}+(k+1) u\right]+O\left(z^{2}\right), \dot{u}=u\left[2 b_{2 k}+(2 k+1) u\right]+O(z) .
$$

This system has two singular points: the point $z=0, u=0$ (which arises only due to the non-regularity of the blowing-up map) and $z=0, u=u_{0}$, where $u_{0}=-2 b_{2 k} /(2 k+1)$ (which is a $2(2 k+1): 1$ resonant node). The system (17) has the 1 -parameter family of integral curves

$$
\left(u-u_{0}\right)\left(u / z^{2}\right)^{2 k-1}(1+O(z))-\delta(1+O(z)) \ln \left(z^{2} / u\right)=\text { const }
$$

or

$$
y^{2}-u_{0} x^{2 k+1}+\ldots+\mu+d \ln x+o(1)=0 .
$$

The term $d \ln x$ corresponds to the term $\delta \tilde{y}^{N} \ln \tilde{y}$ in the normal form (15) near the point $\left(0, u_{0}\right)$.

The point $(0,0)$ is $2: 1$ resonant, but its normal form does not contain the resonant term because it arises from squeezing the line $y=0$, near which all trajectories are analytic.

(b) When $n=2 k-1>2 m+1$, then we use the blowing-up

$$
z=1 / x, u=y / x^{k}
$$

which gives

$$
\dot{z}=z u, \dot{u}=b_{2 k-1}+k u^{2}+b_{2 k-2} z+\ldots+\left(b_{k+m}+a_{m} u\right) z^{k-m}+\ldots .
$$

This system has two singular points $z=0, u= \pm u_{0}= \pm \sqrt{-b_{2 k-1} / k}$ which are $2 k: 1$ resonant. In neighbourhoods of each of these points we have the situation as in Theorem 3.5 (c). So, $u-u_{ \pm}+O(z)=\nu z^{2 k}(1+O(z))+d^{ \pm} z^{2 k}(1+O(z)) \ln z$, which means that $y-u_{ \pm} x^{k}+O\left(x^{k-1}\right)=\nu x^{-k}(1+o(1))+d^{ \pm} x^{-k}(1+o(1)) \ln x$. 
3.8. Proof of Lemma 3.3. If $n=2 m+1$, then we use the blowing-up

$$
z=1 / x, u=y / x^{m+1}
$$

and obtain

$$
\dot{z}=u z, \dot{u}=(m+1) u^{2}+a_{m} u+b_{2 m+1}+O(z)
$$

with two singular points $z=0, u=u_{1,2}$. The ratios of eigenvalues

$$
\lambda_{1,2}=u_{1,2} /\left(2(m+1) u_{1,2}+a_{m}\right.
$$

at these points satisfy (13) from Lemma 3.3. We have $\lambda_{1}+\lambda_{2}=1 /(m+1)$.

Now the result follows from Theorem 3.5.

Remark. It is not clear that the constants $d$ and $d^{ \pm}$, defined in Lemmas $3.1-3.3$, are nontrivial (e.g. are nonzero for a generic system from $\mathcal{A}_{m, n}$ ). Notice that the class of Liénard systems is very restricted. We do not answer this question in this paper.

Probably the behaviour of phase curves of the Liénard system near infinity is analogous to the phase portrait of an analytic system near a nilpotent singular point: $\dot{x}=y+\ldots, \dot{y}=\ldots$ There after suitable resolution of the singularity one obtains an exceptional divisor with singular points of resonant type (see [2]). However, the problem of analytic normal form for such singularities is not solved. The generalized cusp case is completely investigated in [5].

\section{THE LOCAL FIRST INTEGRAL}

In this section we prove the results about non-existence of $d$-fold invariant algebraic curves, $d>2$. This allows us to finish the proof of point (d) of Theorem 1 (non-existence of $d$-fold limit cycles, $d>2$ ) and of Theorem 2. By the way we obtain the proof of the result of Odani.

Unfortunately, the method of Odani (estimation of degrees) does not work here. (The author tried it without success.) We use another method, based again on the analysis of the system near the line at infinity. We shall show the existence of a first integral of some special kind.

4.1. Proposition. Consider the analytic system

$$
\begin{gathered}
\dot{x}=a(x)+a_{1}(x) y+a_{2}(x) y^{2}+\ldots, \\
\dot{y}=b(x) y+b_{2}(x) y^{2}+b_{3}(x) y^{3}+\ldots,
\end{gathered}
$$

defined in a neighbourhood of the line $\{y=0\}=\mathbb{C} P^{1} \subset \mathbb{C} P^{2}$ (also near the point (1:0:0) at infinity). Then there are two possibilities:

(i) There is a formal local Darboux integral

$$
H=y e^{g(x)}\left[\prod_{1}^{r}\left(x-x_{i}\right)^{\alpha_{i}}\right]\left(1+\sum y^{j} w_{j}(x)\right),
$$

where the $x_{i}$ are zeroes of the polynomial $a(x), g$ and the $w_{j}$ are rational functions with poles at the $x_{i}$, and $\alpha_{i} \in \mathbb{C}$.

(ii) There is a positive integer $k$ and a first integral of the local approximate Darboux-Schwartz-Christoffel type

$$
H=y^{-k} F(x, y) e^{g(x)} \prod_{1}^{r}\left(x-x_{i}\right)^{\alpha_{i}}+\int^{x} e^{g(s)} \prod\left(s-x_{i}\right)^{\alpha_{i}-1} P(s) d s+o(1),
$$


where $F(x, y)=1+O(y)$ is an analytic function near $y=0, P$ is a polynomial, $g$ is a rational function and $\alpha_{i} \in \mathbb{C}$.

Proof. We can write the equation for trajectories of (20)

$$
\frac{d y}{d x}=\frac{b}{a}(x) y+c_{2}(x) y^{2}+\ldots,
$$

where the $c_{j}(x)$ are rational functions with poles at $x_{i}$, the zeroes of the polynomial $a(x)$. We seek a first integral of $(22)$ in the form of a series

$$
H=h_{1}(x) y+\sum_{i=2}^{\infty} h_{j}(x) y^{j},
$$

where

$$
\frac{\partial H}{\partial x}+\frac{\partial H}{\partial y} \frac{\partial y}{\partial x}=0
$$

The function $h_{1}(x)$ satisfies the equation

$$
h_{1}^{\prime}+(b / a) h_{1}=0
$$

with the solution $h_{1}=\exp \left(-\int^{x}(b / a)(s) d s\right)$, which after representation of $b / a$ in the form of simple factors $\sum a_{i j}\left(x-x_{i}\right)^{-j}+$ (polynomial) becomes

$$
h_{1}(x)=e^{g(x)} \prod\left(x-x_{i}\right)^{\beta_{i}}
$$

with $\beta_{i} \in \mathbb{C}$, where the $x_{i}$ are zeroes of the polynomial $a(x)$, and a rational function $g(x)$ with poles at $x_{i}$.

For the function $h_{2}$ we get the equation

$$
h_{2}^{\prime}+2(b / a) h_{2}=-c_{2} h_{1},
$$

which we integrate in the form

$$
h_{2}=-h_{1}^{2}(x) \int^{x} h_{1}^{-1}(s) c_{2}(s) d s .
$$

Here we do not fix the lower limit of integrations.

We have two possibilities: either we can choose $h_{2}$ in the form $h_{1}(x) w_{2}(x)$ with rational $w_{2}$, or $h_{2} / h_{1}$ cannot be rational. If $h_{2} / h_{1}$ cannot be rational, then we stop the calculations. If $h_{2} / h_{1}$ is rational, then the constant of integration is defined precisely and we pass to the term $h_{3} y^{3}$.

We have

$$
h_{3}^{\prime}+3(b / a) h_{3}=h_{1}(x) v_{3}(x),
$$

where $v_{3}$ is rational and $h_{3}=-h_{1}^{3}(x) \int^{x} h_{1}^{-2} v_{3}$ is either of the form $h_{1} w_{3}, w_{3}$ rational, or not. If not, then we pass to the terms $h_{4} y^{4}$, etc.

Finally there are two possibilities:

(i) For all $i>1, h_{i} / h_{1}=w_{i}(x)$ is rational and we have the first integral (21).

(ii) There is some smallest $k$ such that the $h_{i} / h_{1}$ are rational for $i<k$ and $h_{k, 0} / h_{1}$ cannot be choosen rational. In this case we change the first integral $H \rightarrow H^{-k}$. One can easily see that we can represent the new integral in the form described in Proposition 4.1.

Remark. One may ask why we have the local Darboux-Schwartz-Christoffel integral in the $1: 1$ resonant node with $H_{0}=x / y-\delta \ln y$. But observe that $H_{0}-\delta H_{0}=x / y-\delta \int d x / x+O(y \ln y)$ is just the integral from Proposition 4.1. 
We apply the integral from Proposition 4.1 to the Liénard system after blowing up the singular point at infinity. We get one of the systems (16), (17), (18), (19) near the line $z=0$.

4.2. Lemma. If $n<2 m+1$, then the system (16) has a local Darboux-SchwartzChristoffel integral of the form

$$
H=\frac{U(u, z)^{\alpha}}{z^{2 m+1-n}}+\psi_{0}(u)+O(z),
$$

where $U(u, z)=u-u_{0}+\frac{1}{m} a_{m-1} z+\ldots+\frac{1}{n-m}\left(a_{n-m-1}+b_{n} / u_{0}\right) z^{2 m+1-n}, \alpha=$ $\frac{2 m+1-n}{m+1}, \psi_{0}(u)=c I\left(1-u / u_{0}\right)$

$$
\begin{gathered}
I(t)=(m+1) \int^{t} \frac{s^{2 m+1-n}}{1-s^{m+1}} d s=\sum_{i=0}^{m+1} \zeta^{-(2 m+1-n) i} \ln \left(1-\zeta^{i} t^{1 /(m+1)}\right), \\
\zeta=e^{2 \pi i /(m+1)}, c=-b_{n} \frac{2 m+1-n}{(m+1)^{2}}\left(-u_{0}\right)^{-(n+1) /(m+1)} .
\end{gathered}
$$

Proof. Representing $b_{j}$ as $\frac{b_{j}}{u_{0}} u-\frac{b_{j}}{u_{0}}\left(u-u_{0}\right)$, we can rewrite the vector field (16) as $V=V_{0}+V_{1}$, where

$$
V_{0}=u\left[z \partial_{z}-\left((m+1)\left(u-u_{0}\right)+a_{m-1} z+\ldots+a_{0} z^{m}+\frac{b_{n}}{u_{0}} z^{2 m+1-n}+\ldots\right) \partial_{u}\right]
$$

is integrable with the first integral $\Phi=\frac{U(u, z)+\ldots}{z^{m+1}}$. Let $H_{0}=\Phi^{\alpha}$; it is approximately the first part of the integral (23). We treat $V_{1}$ as a perturbation of $V_{0}$.

If $\psi_{i}(u)$ are the coefficients before $z^{i}$ in the expansion of $H$, then $\psi_{0}$ satisfies the equation

$$
(m+1) u \psi_{0}^{\prime}+\frac{b_{n}}{u_{0}} \psi_{n-2 m-1}^{\prime}=0,
$$

$\psi_{n-2 m-1}=\left(u-u_{0}\right)^{(2 m+1-n) /(m+1)}$, with the solution as in Lemma 4.2.

Remark. The above form of the approximate first integral agrees with the result of [4], where it was shown that any elementary first integral of the Liénard equation for $m<n<2 m+1$ is of the Darboux-Schwartz-Christoffel type

$$
H=\left[y+p_{2}(x)\right]^{\alpha}+c I\left(\frac{y+p_{2}(x)}{\left(p_{2}-p_{1}\right)(x)}\right)
$$

with ramifications along the curves $y+p_{1}(x)=0$ and $y+p_{2}(x)=0$.

4.3. Lemma. . If $n<2 m+1$, then any invariant algebraic curve is either rational or hyperelliptic (as in Section 2).

Proof. Assume that there is a $d$-fold, $d>2$, invariant curve $\Omega$. Let us look at its intersection points with infinity. When we work with the form (16) then $\Omega$ can intersect the line $z=0$ at two points: $A=(0,0)$ and $B=\left(0, u_{0}\right)$. By Lemma 3.1 only one branch of $\Omega$ can pass through the point $A$ (as the center manifold) and any branch passing through $B$ is of the form

$$
y=M(x)+\mu_{i}+O(1 / x), x \rightarrow \infty,
$$

where $M(x)$ is some fixed polynomial and $\mu_{i}=$ const.

If some irreducible component $\Omega_{0}$ of $\Omega$ does not pass through $A$ (only through $B)$, then we apply the change of variables $(x, y) \rightarrow(x, w), w=y-M(x)$. The local components $w=\mu_{i}+O(1 / x)$ intersect the line at infinity $L_{\infty}=\{(x: w: 0)\}$ 
transversally at the point $(1: 0: 0)(x=\infty, w=0)$. By the Bézout theorem the intersection index of $\Omega_{0}$ with $L_{\infty}$ at $(1: 0: 0)$ is equal to its degree. Now it is easy to see that $\Omega_{0}$ must be a line $w=\mu_{i}$ (perform the analysis in the coordinates $(1 / x, w / x)$ ). One can also see that there can be at most one such line (because $\dot{w}$ is linear in $w$, it also follows from the argument below).

Also when $\Omega_{0}$ passes only through the point $A$, then it is a parametric rational curve.

Therefore, we have to consider the situation when some irreducible component $\Omega_{0}$ of $\Omega$ passes through the both points $A$ and $B$. Using Lemma 4.2, we can assume that near the line $z=0$ there exists the first integral $H$ with a logarithmic singularity at the center manifold $\Gamma_{0}^{0}$ of $A$ and with a root singularity along some trajectory $\Gamma_{\mu_{0}}$ passing through the point $B$. We have $\left.H\right|_{\Gamma_{0}^{0}}=\infty,\left.H\right|_{\Gamma_{\mu_{0}}}=0$. The other local components $\Gamma_{\mu_{i}}, i=1, \ldots, s$, of $\Omega$ are given by the equations $H(z, u)=c_{i}\left(\right.$ or $\left.H^{1 / \alpha}=\mu_{i}\right)$.

Remark. We cannot expect that the first integral is of the form $\varphi^{\alpha}+c I(\varphi / \eta)$ ( $\varphi, \eta$ holomorphic functions), because the holonomy group of the punctured line $X=\{z=0\} \backslash\{A, B\}$ (the image of $\pi_{1}(X)$ in the group of local diffeomorphisms of a local holomorphic disc transversal to $X$ in $\mathbb{C}^{2}$ ) may be complicated (e.g. nonsolvable).

We can assume that the equation for $\Gamma_{0}^{0}$ is

$$
y-P_{n-m}(x)-\phi(x, y)=0, \phi(x, y) \rightarrow 0,
$$

$\phi$ analytic; the equation for $\Gamma_{\mu_{0}}$ is

$$
y-M_{m+1}(x)-\mu_{0}-\psi(x, y), \psi \rightarrow 0,
$$

$\psi$ analytic, and the equations for $\Gamma_{\mu_{i}}, i=1, \ldots, s$, are

$$
y=M_{m+1}(x)-\mu_{i}-\eta_{i}(x, y),
$$

where $\eta_{i} \sim x^{n-m} I\left(1-y /\left(u_{0} x^{m+1}\right)\right)+\ldots$ are not analytic.

The polynomial $K(x, y)$ defining the curve $\Omega$ is the product of these functions. But such a product cannot be a polynomial for $s \geq 1$ because it contains the term $x^{s(n-m)} I^{s}$.

This completes the proof of Lemma 4.3 and of Theorem 2 for $n<2 m+1$. Also the $d$-fold, $d>2$, limit cycles are eliminated in this case.

4.4. Lemma. If $n=2 k-1>2 m+1$, then the system (18) has local DarbouxSchwartz-Christoffel integral (not Darboux) of the type

$$
H=\frac{U(u, z)^{\alpha}}{z^{k-m}}-\alpha \int^{u}\left(k s^{2}+b_{2 k-1}\right)^{\alpha-2}\left(b_{k+m} s+a_{m} s^{2}\right) d s+O(z),
$$

where

$$
U(u, z)=\frac{u^{2}}{2}+\frac{b_{2 k-1}}{2 k}+\frac{b_{2 k-2} z}{2 k-1}+\ldots+\frac{b_{k+m+1} z^{k-m-2}}{k+m+2}, \alpha=\frac{k-m-1}{2 k} .
$$

An analogous lemma holds in the case of even $n=2 k>2 m+1$.

Proof. Note that if $f_{m}(x) \equiv 0$, then the system (1) is Hamiltonian. So, up to terms of order $O\left(x^{k+m}\right)$, we have the following expansion for the first integral:

$$
H=\frac{1}{2} y^{2}+\frac{b_{2 k-1}}{2 k} x^{2 k}+\frac{b_{2 k-2}}{2 k-1} x^{2 k-1}+\ldots+\frac{b_{k+m+1}}{k+m+2} x^{k+m+2}+\ldots
$$


Using the notations from Section 3.7, we get

$$
\begin{aligned}
H= & \frac{u^{2} / 2+b_{2 k-1} /(2 k)}{z^{2 k}}+\frac{b_{2 k-2}}{(2 k-1) z^{2 k-1}} \\
& +\ldots+\frac{b_{k+m+1}}{(k+m+2) z^{k+m+2}}+\frac{h(u)}{z^{k+m}}+\ldots .
\end{aligned}
$$

The function $h(u)$ satisfies the equation

$$
-(k+m+1) u h+\left(b_{2 k-1}+k u^{2}\right) h^{\prime}+u\left(b_{k+m}+a_{m} u\right)=0
$$

(see (18)), which gives

$$
h(u)=-\left(k u^{2}+b_{2 k-1}\right)^{1-\alpha} \int^{u}\left(k s^{2}+b_{2 k-1}\right)^{\alpha-2}\left(b_{k+m} s+a_{m} s^{2}\right) d s .
$$

This integral is non-elementary (i.e. not of Darboux type) because

$$
\int_{-u_{0}}^{u_{0}}\left(k s^{2}+b_{2 k-1}\right)^{\alpha-2} s^{2} d s \neq 0
$$

and $u_{0}=\sqrt{-b_{2 k-1} / k}$ for $b_{2 k-1} \neq 0$.

4.5. Lemma. If $n>2 m+1$, then any invariant algebraic curve is either rational or hyperelliptic (as in Section 2).

Proof. We present the proof only for the case of odd $n=2 k-1$.

If $\Omega$ is an invariant algebraic curve, then its intersections with infinity are at the points $A_{ \pm}: z=0, u= \pm u_{0}$. Repeating the proof of Lemma 4.4, we can assume that two of the local components of $\Omega$ form the root singularities of the first integral (24); $u= \pm u_{0}+O(z)$ (or $y-P_{k}^{ \pm}(x)-\phi^{ \pm}(x, y)=0, \phi^{ \pm} \rightarrow 0, \phi^{ \pm}$analytic). Other components have the expansions with non-analytic terms: $y-R_{i}(x)+\eta_{i}(x, y)=0$, $\eta_{i} \sim x^{k+m} h\left(y / x^{k}\right)$. The product of such functions cannot give a polynomial.

Similar arguments work for even $n$.

So, if $n>2 m+1$, then Theorem 2 holds.

4.6. Lemma. Let $n=2 m+1$.

(a) If $\lambda_{1}, \lambda_{2} \notin \mathbb{Q}$ (see Lemma 3.3), then any invariant algebraic curve of the system (1) is of the form $y=P_{m+1}(x)$ or of the form $\left(y+P_{m+1}\right)^{2}=Q(x)$.

(b) If $\lambda_{1,2} \in \mathbb{Q}$, then either we have the situation as in case (a) or there is an invariant $d$-fold algebraic curve, $d>2$, the singular points $\left(0, u_{1,2}\right)$ are formally linearizable and there is a formal local Darboux integral in the system (19).

Proof. If $\lambda_{1,2} \notin \mathbb{Q}$, then by Lemma 3.3 there exist exactly two analytic trajectories going to infinity. Hence, the only invariant algebraic curves are one- or two-fold.

If $\lambda_{1,2} \in \mathbb{Q}$ and there is a $d$-fold curve $\Omega, d>2$, then one of the singular points of the system (19) is a resonant and linearizable node through which at least two branches of $\Omega$ pass. We look at the local first integral near the line $z=0$ and we repeat the analysis from the proofs of Lemma 4.3 and Lemma 4.5. One sees that the integral must be of formal local Darboux type. This implies that both singular points are formally linearizable.

This completes the proof of Lemma 4.6 and of Theorem 2 .

Remark. If there is a formal local Darboux first integral, then the local holonomy group associated with the holomorphic foliation near the line $y=0$ is formally 
abelian. Maybe, in such case there is a local analytic Darboux first integral (i.e. of the form $\prod f_{i}^{a_{i}}$ with analytic functions $\left.f_{i}(x, y)\right)$ and the holonomy group is abelian.

\section{Density of the Set of Systems Without InVARIANT ALGEBRAiC CURVE}

Here we prove point (b) of Theorem 1.

In the case $m<n<2 m+1$ (by Lemma 4.3) we have to show that the systems with rational invariant curves and systems with hyperelliptic invariant curves form thin subsets of $\mathcal{A}_{m, n}$. Because they form algebraic subsets, it is enough to calculate their dimensions.

If there is an invariant algebraic curve of the form $y=P_{n-m}(x)$, then $g_{n}=$ $-\left(f_{m}+P_{n-m}^{\prime}\right) P_{n-m}$ (see Section 2.1). We get the subvariety $\Sigma$ in $\mathcal{A}_{m, n}$ (parametrized by $f_{m}$ and $\left.P_{n-m}\right)$ consisting of systems with such invariant curves. Its dimension is $(m+1)+(n-m+1)=n+2$, which is smaller than $\operatorname{dim} \mathcal{A}_{m, n}=m+n+2$ for $m>0$.

If there is an invariant algebraic curve of the form $y=P_{m+1}(x)$, then $P_{m+1}=$ $-F+Q_{n-m}, F^{\prime}=f_{m}$ (see Section 2.1). The corresponding subvariety is parametrized by $f_{m}$ and $Q_{n-m}$ and is of codimension $m$.

If there is an invariant hyperelliptic curve, then $P_{m+1}=a \prod\left(x-x_{i}\right)^{m_{i}}, Q=$ $b \prod\left(x-x_{i}\right)^{n_{i}}$ with $2 m-n$ restrictions onto $x_{i}$ (see Section 2.2). We see that the set of systems with such an invariant curve is parametrized by the discrete parameters $r, m_{i}, n_{i}$ and $r+2-(2 m-n)(\leq n+3-m)$ continuous parameters $a, b, x_{i}$. This set forms a finite union of algebraic subvarietes of codimensions $\geq 2 m-1>0$ for $m>0$.

In the case $n>2 m+1$ we repeat the arguments from the case $n<2 m+1$. If $n=2 k-1$, then there is a subvariety consisting of systems with a rational invariant curve of the form $y=P_{k}(x)$ and parametrized by $P_{k}$ and $f_{m}$. Its codimension is $k-1>0$. The subvarietes corresponding to hyperelliptic algebraic curves are parametrized by $a, b, x_{i}$ (without restrictions) and have dimension $\leq m+3$, which is smaller than $\operatorname{dim} \mathcal{A}_{m, n}>3 m+3$.

In the case $n=2 m+1$ we can assume that $\lambda_{1,2} \notin \mathbb{Q}_{+}$in Lemma 3.4. Hence by Lemma 4.6 the problem becomes the same as the analogous problem in the case $n>2 m+1$. The proof is also the same for $m>0$. (If $m=0$ then the system is linear and has an invariant line).

\section{The EXAMPLE of YABLONSKY}

In [7] Yablonsky constructed an example of quadratic system with an algebraic limit cycle. To implement his method, consider the 12-parameter family of quadratic systems

$$
\dot{x}=F_{2}(x, y), \dot{y}=G_{2}(x, y) .
$$

We seek a limit cycle in the form

$$
y=w_{1}(x) \sqrt{P_{2}(x)}+T_{2}(x),
$$

where $w_{1}=\alpha_{0} x+\alpha_{1}, P_{2}=-x^{2}+2 p x+q^{2}, T_{2}=\beta_{0} x^{2}+\beta_{1} x+\beta_{2}$. In order to construct a limit cycle, we substitute (26) into (25) and find a series of very complex conditions for invariance of the curve (26). Next we check that (26) is a limit cycle for a suitable choice of parameters.

Note that (26) describes a rational degeneration of the elliptic curve $\left(y-T_{2}\right)^{2}=$ $w_{1}^{2} P_{2}$ of fourth degree with one singular point $\left(x_{0}, y_{0}\right), w_{1}\left(x_{0}\right)=0, y_{0}=T_{2}\left(x_{0}\right)$, a 
simple double point. In a footnote in his paper, Yablonsky remarks that he can do the same with the smooth elliptic curve $y=\sqrt{R_{4}(x)}+T_{2}(x), \operatorname{deg} R_{4}=4, \operatorname{deg} T_{2}=$ 2. As we shall see, this cannot hold.

We begin the analysis of elliptic limit cycles for quadratic systems with the following property (see [8], Lemma 7).

6.1. Lemma. (a) If $K(x, y)=0$ is a smooth irreducible invariavt algebraic curve for a polynomial planar vector field $V(x, y)$, then there exist a polynomial $h(x, y)$ and a polynomial vector field $W(x, y)$ such that

$$
V=h X_{K}+K W
$$

where $X_{K}=\frac{\partial K}{\partial y} \frac{\partial}{\partial x}-\frac{\partial K}{\partial x} \frac{\partial}{\partial y}$ is a Hamiltonian vector field.

(b) If the above curve $K(x, y)=0$ has singular points $p_{1}, \ldots, p_{s}$ of the double point type and no other singularities, then for any polynomial $S(x, y)$ such that $S\left(p_{i}\right)=0$ there is the representation

$$
S V=h X_{K}+K W
$$

6.2. Proposition. If $K(x, y)=(y-P(x))^{2}-Q(x)=0, \operatorname{deg} P \leq 2, \operatorname{deg} Q \leq$ $4, \max (\operatorname{deg} P,(\operatorname{deg} Q) / 2)=2$, is a smooth curve, then it cannot form a limit cycle for any quadratic vector field.

Proof. We have

$$
X_{K}=\left(\begin{array}{c}
2(y-P) \\
2(y-P) P^{\prime}+Q^{\prime}
\end{array}\right)=\left(\begin{array}{l}
\Phi_{1} \\
\Phi_{2}
\end{array}\right) .
$$

The curve $K=0$ has two local branches near infinity.

Assume that one of them is $y=\phi_{1}(x) \sim C x^{2}$. We have

$$
\left.X_{K}\right|_{y=\phi_{1}(x)} \sim\left(\begin{array}{c}
x^{2} \\
x^{3}
\end{array}\right) .
$$

If

$$
V=\left(\begin{array}{c}
\cdots+a x^{2}+b x y+c y^{2} \\
\cdots+d x^{2}+e x y+f y^{2}
\end{array}\right)
$$

then

$$
\left.V\right|_{y=\phi_{1}(x)} \sim\left(\begin{array}{c}
a x^{2}+b C x^{3}+c C^{2} x^{4} \\
d x^{2}+e C x^{3}+f C^{2} x^{4}
\end{array}\right) .
$$

If the curve $K=0$ contains a limit cycle, then we have the representation (27) with $W \not \equiv 0$. Restricting it to the branch $y=\phi_{1}(x)$ and using (29) and (30), we get that $c=0$ and $\mid h\left(x, \phi_{1}(x) \mid<O(x)\right.$. Thus $h(x)=A x+B$. The first components of $V(x, y)$ and of $h(x, y) X_{K}(x, y)$ are linear in $y$. Therefore, the first component of $W(x, y)=\left(W_{1}, W_{2}\right)$ is zero. But then $h(x, y) P(x)$ is quadratic and either

(i) $h(x, y)=$ const or (ii) $P(x)$ is linear.

In case (i), $W_{2}(x, y) \equiv$ const, $\Phi_{2}(x, y)$ is cubic in $x$, and then $\left(P^{2}-Q\right)(x)$ is at most cubic in $x$. This implies that $\Phi_{2}(x, y)$ is at most quadratic in $x$, and then $\left(P^{2}-Q\right)(x)$ is quadratic in $x$. Next, the coefficient before $y$ in $\Phi_{2}(x, y)$ is $2 P^{\prime}(x)$ and is linear, whereas $K(x, y) W_{2}(x, y)$ contains $2 y P(x)$. So, $P(x)$ is linear and $Q(x)$ is quadratic.

In case (ii), $P(x)$ is linear and we can put $h(x, y)=x$ and $K(x, y)=y^{2}-Q(x)$, so that $h X_{K}=(2 x y, x Q(x))$. This also leads to $W_{1}(x y) \equiv 0, W_{2}(x, y) \equiv$ const and 
$\operatorname{deg}\left(x Q^{\prime}(x)+W_{2} Q(x)\right)=2$. But then $W_{2}=4$ and $Q(x)=A x^{4}+B x^{2}+C x+D$. We get

$$
\dot{x}=2 x y, \dot{y}=-4 D-3 C x-2 B x^{2}-4 y^{2} .
$$

This system has first integral $H=x^{-4}\left(y^{2}+B x^{2}+C x+D\right)$ and no limit cycles.

Remark. Repeating the arguments from the above proof, one can easily show that a quadratic system cannot have 2-fold limit cycle in an elliptic curve $y^{2}=A x^{3}+$ $B x+C, A \neq 0$.

6.4. Limit cycles at a singular 2-fold curve. Here we give our presentation of the example of Yablonsky. We hope that our analysis is simpler that that from [7].

We choose

$$
K=\left[y-\left(a x^{2}+b x+c\right)\right]^{2}-x^{2}\left(d x^{2}+e x+f\right) .
$$

We put $S(x, y)=x$ in (26). Then we have $\left.h\right|_{K=0} \sim x^{2}$, and hence $h(x, y)=$ $A y+B x^{2}+C x+D$. Thus $W_{1}=E=$ const, $W_{2}=F x+G$.

Putting $x=0$ in (28), we get

$$
\begin{aligned}
& 2(y-c)(A y+D)+E(y-c)^{2}=0, \\
& 2(y-c) b(A y+D)+G(y-c)^{2}=0,
\end{aligned}
$$

so that $E=-2 A, D=-A c, G=-2 A b$.

Next, the first

$$
2\left(y-a x^{2}-b x-c\right) h(x, y)+E K(x, y)
$$

and the second

$$
\left[2\left(y-a x^{2}-b x-c\right)(2 a x+b)+4 d x^{3}+3 e x^{2}+2 f x\right] h(x, y)+(F x+G) K(x, y)
$$

components of the right hand side of (28) do not contain the terms $x^{5}, x^{4}, x^{3} y$. This gives the equations

$$
\begin{gathered}
-2 a B-2 A\left(a^{2}-d\right)=0, \\
4\left(d-a^{2}\right) B+F\left(a^{2}-d\right)=0, \\
3(e-2 a b) B+4\left(d-a^{2}\right) C-2 A b\left(a^{2}-d\right)+F(2 a b-e)=0, \\
4 a B+4\left(d-a^{2}\right) A-2 a F=0 .
\end{gathered}
$$

From the first three equations we find that $B=A\left(d-a^{2}\right) / a, F=4 A\left(d-a^{2}\right) / a, C=$ $(2 a b-e) /(4 a)$, and the forth equation also holds.

We see that such systems $V=\left[h X_{K}+K W\right] / x$ are parametrized by the 7 parameters $a, b, c, d, e, f, A$.

Now we show that if the parameters are chosen suitably then the oval $\gamma$ of the curve $K=0$ is a limit cycle.

Let us look at the singular point $x=0, y=c$ with the matrix $M$ of its linearization. One can easily calculate that $\operatorname{tr} M=2 f$ and $\operatorname{det} M=-2 f(b+C)(b+2 C), C=$ $b / 2-e /(4 a)$. We see that the point $(0, c)$ can be of focus type.

Since the quadratic systems with non-isolated periodic solutions have a center, we need only eliminate the situation with a center and a focus. 
The quadratic systems with center have been classified (see [9] for example). There is only one case with a center and a focus; it is the class of Lotka-Volterra systems $Q_{3}^{L V}$ with a pair of imaginary invariant lines. The first integral is of the form $H=\left(P_{1}+i Q_{1}\right)^{\alpha+i \beta}\left(P_{1}-i Q_{1}\right)^{\alpha-i \beta} R_{1}, \beta \neq 0, Q_{1} \not \equiv 0$, where $P_{1}, Q_{1}, R_{1}$ are linear real functions. The only algebraic level curves of such an integral are the three lines $P_{1}=0, Q_{1}=0, R_{1}=0$. Of course, $K=0$ is not of this type.

\section{ACKNOWLEDGEMENTS}

The author thanks K. Odani for sending him the preprint [4]. It helped to avoid some mistakes. The remarks of the referee helped to improve the presentation of the text.

\section{REFERENCES}

[1] Arnold V. I. and Il'iashenko Yu. S. Ordinary differential equations, Itogi Nauki i Tekhniki: Sovremennye Problemy Mat.: Fundamental'nye Napravleniya, vol. 1, VINITI, Moscow, 1985, pp. 7-149; English transl. in Encyclopaedia of Math. Sci., vol. 1 [Dynamical Systems, 1], Springer-Verlag, Berlin, 1988. MR 87e:34049

[2] Cerveau D. and Moussu R. Groupes d'automorphismes de $(\mathbb{C}, 0)$ et équations différentielles $y d y+\ldots=0$. Bull. Soc. Math. France 116 (1988) 459-488. MR 90m:58192

[3] Odani K. The limit cycle of the van der Pol equation is not algebraic. J. Diff. Equat. 115 (1995) 146-152. MR 95i:34051

[4] Odani K. The integration of polynomial Liénard system in elementary functions (preprint). (1995).

[5] Stróżyna E. and Żołądek H. The analytic normal form for the nilpotent singularity (preprint). (1996).

[6] Wilson J. C. Algebraic periodic solutions of Liénard equations. Contrib. to Diff. Equat. 3 (1964) 1-20. MR 28:3203

[7] Yablonsky A. I. On limit cycles of certain differential equation. Diff. Uravneniya 2 (1966), 335-344; English transl. in Differential Equations 2 (1966). MR 33:1538

[8] Żołądek H. The classification of reversible cubic systems with center. Topol. Methods in Nonlin. Anal. 4 (1994) 79-136. MR 96m:34057

[9] Żołądek H. Quadratic systems with center and their perturbations. J. Diff. Equat. 109 (1994) 223-273. MR 95b:34047

Institute of Mathematics, University of WarsaW, Banacha 2, 02-097 WarsaW, Poland E-mail address: zoladek@mimuw.edu.pl 